\title{
Restrictions to neutron star models based on twin-peak quasi-periodic oscillations
}

\section{Gabriel Török, Martin Urbanec, Kateřina Goluchová, Pavel Bakala, Eva Šrámková and Zdeněk Stuchlík}

Institute of Physics, Faculty of Philosophy and Science, Silesian University in Opava, Bezručovo nám. 13, CZ-74601 Opava, Czech Republic

email: gabriel.torok@gmail.com

\begin{abstract}
In a series of works - Török et al. (2010, 2012a) and Urbanec et al. (2010a) - we explored restrictions to neutron star properties that are implied by various models of twin-peak quasi-periodic oscillations. Here we sketch an attempt to confront the obtained mass-angularmomentum relations and limits on neutron star compactness with the parameters estimated by assuming various equations of state and the spin frequency of the atoll source $4 \mathrm{U}$ 1636-53.
\end{abstract}

Keywords. X-rays: binaries; stars: neutron; stars: fundamental parameters; stars: rotation

\section{Introduction}

Twin-peak quasi-periodic oscillations (kHz QPOs) appear in the X-ray power-density spectra of several accreting low-mass neutron star (NS) binaries. Frequencies of these QPOs follow correlations specific for a given source (see the left panel of Figure 1 for illustration). Most QPO models relate the observed frequencies to frequencies of the orbital motion inside an inner part of the accretion disc.

The consideration of various orbital QPO models for the NS sources data results in specific mass-angular-momentum $(M-j)$ relations rather than in preferred combinations of $M$ and $j$ (e.g., Török et al. 2012b). For the atoll source $4 \mathrm{U} 1636-53$, there is a good evidence on the NS spin frequency based on the X-ray burst measurements, Strohmayer \& Markwardt (2002). Thus, one can in principle infer the angular momentum $j$ and remove the $M-j$ degeneracies related to the individual twin-peak QPO models.

\section{Our approach}

We calculate $\chi^{2}$ maps resulting from the fitting of the $4 \mathrm{U} 1636-53$ data for various twin-peak QPO models (RP - Stella \& Vietri, 1999, Stella et al., 1999; RP1 - Bursa, 2005; RP2 - Török et al., 2010; WD - Kato, 2001; TD - Čadež et al., 2008). These maps are compared to the $M-j$ relations calculated from several NS equations of state (EoS) assuming that the spin frequency is either $290 \mathrm{~Hz}$ or $580 \mathrm{~Hz}$ (from one or two hot-spot models for the X-ray bursts).

For the QPO models we (yet) assume that the influence of the NS oblateness related to the quadrupole moment $q$ is low and it is $q / j^{2} \sim 1$. In the calculations of the NS models we use the geometry of the Hartle-Thorne spacetime (Hartle \& Thorne, 1968) and utilize the set of various EoS. These are namely: SLy 4 - Rikovska-Stone et al. (2003); APR - Akmal et al. (1998); AU-WFF1, UU-WFF2 and WS-WFF3 - Wiringa et al. (1988), Stergioulas \& Friedman (1995). Related details and further references are given in Urbanec et al. (2010a,b). 

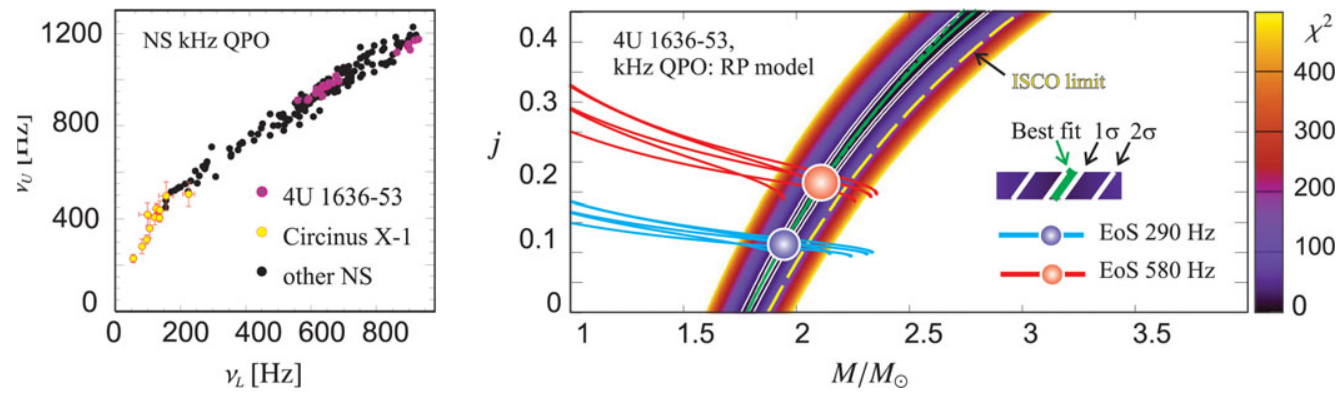

Figure 1. Frequencies of the twin-peak QPOs in various NS sources. Right: The $\chi^{2}$ map of the $\mathrm{RP}$ model vs. the NS EoS. The $\chi^{2}$ map results from the fits of the RP model to the $\mathrm{kHz} \mathrm{QPO}$ data of $4 \mathrm{U}$ 1636-53. The NS EoS are assumed for the rotational frequency inferred from the $\mathrm{X}$-ray burst measurements. The green line indicates the best QPO $\chi^{2}$ for a fixed $M$. The white lines indicate the corresponding $1 \sigma$ and $2 \sigma$ confidence levels. The dashed-yellow line indicates a simplified estimate on the upper limits on $M$ and $j$ assuming that the highest observed upper QPO frequency in $4 \mathrm{U} 1636-53$ is associated to the innermost stable circular orbit (ISCO).

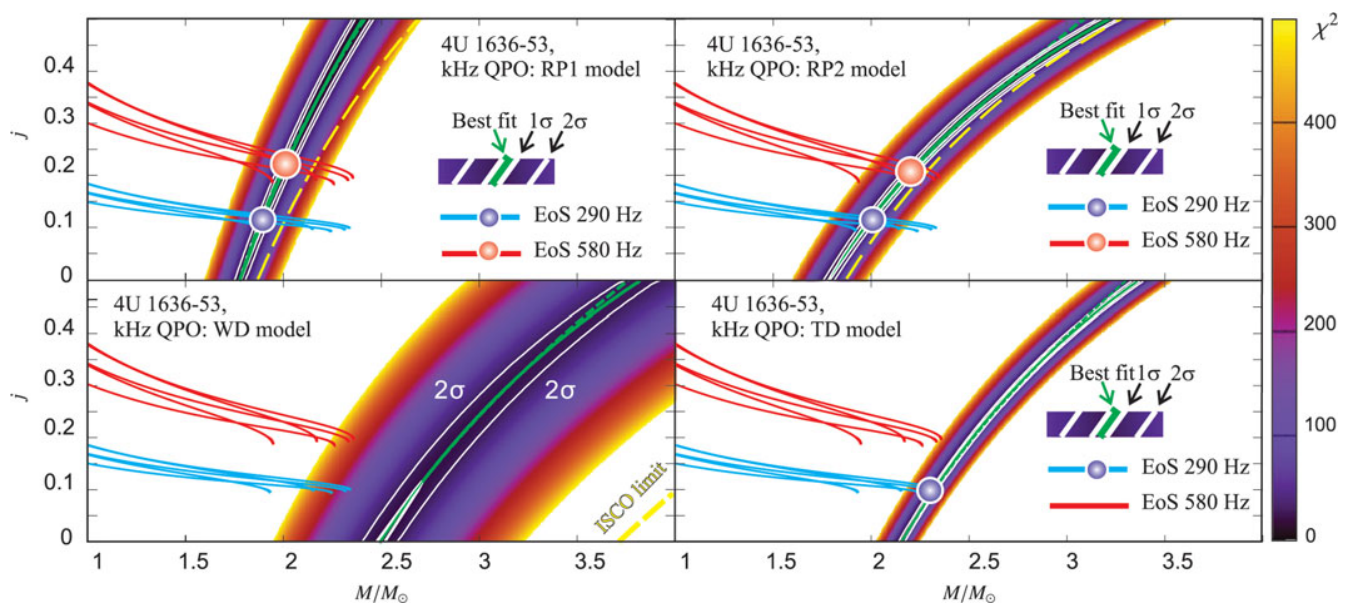

Figure 2. Mass-angular-momentum relations and limits on the NS compactness (the same as in the right panel of Figure 1, but for several other models).

In the right panel of Figure 1 we illustrate the potential of such approach in the case of the relativistic precession (RP) QPO model. Several other models are considered in Figure 2. The inferred NS parameters are summarized in Table 1.

\section{Conclusions}

The presented partial results come from the work in progress and the final assessment requires to complete a fully self-consistent consideration of the quadrupole moment influence. Nevertheless, each application of the concrete EoS clearly removes the degeneracy in mass and angular momentum determined from the QPO models. Moreover, the applied NS EoS seems to be compatible only with some of the considered QPO models and vice versa. 
Table 1. Results for geodesic QPO models. Symbols $\nu_{K}, \nu_{\mathrm{r}}$ and $\nu_{\theta}$ denote the Keplerian and the epicyclic orbital frequencies.

\begin{tabular}{|c|c|c|c|c|}
\hline & & Atoll Sour & e $4 U$ 1636-5 & \\
\hline Model & $M(290 \mathrm{~Hz})$ & $j(290 \mathrm{~Hz})$ & | $M(580 \mathrm{~Hz})$ & $j(580 \mathrm{~Hz})$ \\
\hline $\begin{array}{l}\text { RP: } \quad \nu_{L}=\nu_{K}-\nu_{\mathrm{r}}, \quad \nu_{U}=\nu_{K} \text {. } \\
\text { Modes of relativistic epicyclic motion of blobs } \\
\text { at various radii } r \text { in the accretion disc. }\end{array}$ & $1.9 M_{\odot}$ & 0.11 & $2.1 M_{\odot}$ & 0.21 \\
\hline $\begin{array}{l}\text { TD: } \nu_{L}=\nu_{K}, \quad \nu_{U}=\nu_{K}+\nu_{\mathrm{r}} \text {. } \\
\text { The QPOs generated by a tidal disruption } \\
\text { of large accreting inhomogeneities. }\end{array}$ & $2.3 M_{\odot}$ & 0.10 & - & - \\
\hline $\begin{array}{l}\text { RP1: } \quad \nu_{L}=\nu_{K}-\nu_{\mathrm{r}}, \quad \nu_{U}=\nu_{\theta} . \\
\text { Non-axisymmetric disc-oscillation modes whose } \\
\text { frequencies are similar to the frequencies } \\
\text { predicted by the RP model when } j \sim 0 \text {. }\end{array}$ & $1.8 M_{\odot}$ & 0.11 & $2.0 M_{\odot}$ & 0.21 \\
\hline $\begin{array}{l}\mathrm{RP} 2: \quad \nu_{L}=\nu_{K}-\nu_{\mathrm{r}}, \quad \nu_{U}=2 \nu_{K}-\nu_{\theta} \text {. } \\
\text { Non-axisymmetric disc-oscillation modes whose } \\
\text { frequencies are similar to the frequencies } \\
\text { predicted by the RP model when } j \sim 0 \text {. }\end{array}$ & $2.0 M_{\odot}$ & 0.11 & $2.2 M_{\odot}$ & 0.20 \\
\hline $\begin{array}{l}\text { WD: } \quad \nu_{L}=2\left(\nu_{K}-\nu_{\mathrm{r}}\right), \quad \nu_{U}=2 \nu_{K}-\nu_{\mathrm{r}} \text {. } \\
\text { Another specific non-axisymmetric modes } \\
\text { of accretion disc-oscillations. }\end{array}$ & - & - & - & - \\
\hline
\end{tabular}

\section{Acknowledgements}

The reported work has been supported by the Czech research grants GACR 209/12/P740, MSM 4781305903 and the project CZ.1.07/2.3.00/20.0071 - "Synergy" supporting international collaboration of the Institute of Physics at SU Opava. The authors further acknowledge the internal grant of the Silesian University in Opava, SGS/1/2010.

\section{References}

Akmal, A., Pandharipande, V. R., \& Ravenhall, D. G. 1998, Phys. Rev. C, 58, 1804

Bursa, M. 2005, In Proceedings of RAGtime 6/7, Opava pp. 39

Čadež, A., Calvani, M., \& Kostić, U. 2008, A\& $A$, 487, 527

Hartle, J. B. \& Thorne, K. S. 1968, ApJ, 153, 807

Kato, S. 2001, PASJ, 53, 1

Rikovska-Stone, J., Miller, J. C., Koncewicz, R., Stevenson, P. D., \& Strayer, M. R. 2003, Phys. Rev. $C, 68$

Stella, L. \& Vietri, M. 1999, Phys. Rev. Lett., 82, 17

Stella, L., Vietri, M., \& Morsink, S. M. 1999, ApJ, 524, L63

Stergioulas, N. \& Friedman, J. L. 1995, ApJ, 444, 306

Strohmayer, T. E. \& Markwardt, C. B. 2002, ApJ, 577, 337

Török, G., Bakala, P., Šrámková, E., Stuchlík, Z., \& Urbanec, M. 2010, ApJ, 714, 748

Török, G., Bakala P., Šrámková E., Stuchlík Z., Urbanec M., Goluchová K. 2012a, accepted for publication in ApJ

Török, G., Bakala P., Šrámková E., Stuchlík Z., Urbanec M., Goluchová K. 2012b, Procs. of IAU Symposium 290

Urbanec, M., Török, G., Šrámková, E., Čech, P., Stuchlík, Z., \& Bakala, P. 2010a, A\&\&A, 522, 72

Urbanec, M., Běták, E., \& Stuchlík, Z. 2010b, Acta Astronomica, 60, 149

Wiringa, R. B., Fiks, V., \& Fabrocini, A. 1988, Phys. Rev. C, 38, 1010 